\title{
As Contribuições da Personalidade e dos Eventos de Vida para o Bem-Estar Subjetivo
}

\author{
Carla Woyciekoski ${ }^{1}$ \\ Jean Carlos Natividade \\ Claudio Simon Hutz \\ Universidade Federal do Rio Grande do Sul
}

\begin{abstract}
RESUMO - Este estudo teve por objetivo verificar o poder preditivo de características de personalidade e eventos de vida sobre o bem-estar subjetivo (BES). Participaram 274 universitários, 69\% mulheres. Os resultados mostraram neuroticismo e extroversão como as principais variáveis explicativas do BES. Ainda, seis eventos de vida contribuíram com a explicação do BES, por exemplo, "término de namoro" mostrou-se variável preditiva positiva de Afeto positivo;"problemas financeiros" explicou negativamente a Satisfação de Vida. Os resultados sugerem que, embora as pessoas possam avaliar suas vidas como mais positivas e experienciar afetos mais positivos em função de suas características de personalidade, é pertinente adotar no campo de estudos do BES uma abordagem interacionista, integrando fatores intrínsecos, como personalidade, e extrínsecos, como eventos de vida.
\end{abstract}

Palavras-chave: bem-estar subjetivo, personalidade, eventos de vida

\section{Contributions of Personality and Life Events to the Subjective Well-Being}

\begin{abstract}
The aim of this study was to investigate the contributions of personality traits and life events as predictors of subjective well-being (SWB). Therefore, 274 students were participants, $69 \%$ women. Results showed neuroticism and extraversionas the main predictors of SWB. Furthermore, six life events contributed to the explanation of SWB, for example, "relationship breakup" showed was a positive predictor of PositiveAffect; and "financial problems" predicted Life Satisfaction negatively. The results suggest that although people can evaluate their lives as more positive and experience more positive emotions depending on their personality traits, it is pertinent to adopt an integrative approach in the field of SWB, incorporating intrinsic factors such as personality, as well as extrinsic factors such as life events.
\end{abstract}

Keywords: subjective well-being, personality, life events

A felicidade é frequentemente apontada como uma meta na vida de muitas pessoas. Não coincidentemente, a busca por entendê-la temdespertado o interesse de pensadores ao longo dos séculos. O seu estudo científico compreende esforços no sentido da caracterização desse sentimento, bem como,da identificação de suas causas e seus efeitos na subjetividade e na vida das pessoas. Diener (1984) desenvolveu estudos sobre esse tema e utilizou o termo Bem-Estar Subjetivo (BES) como um conceito científico relacionado à felicidade. Desde então, o BES tem ganhado destaque na pesquisa, passando a ser estudado e medido de forma sistemática.

O BES é considerado um fenômeno amplo que inclui respostas emocionais e julgamentos globais sobre a satisfação com a vida (Diener, 1984; Diener, Suh, Lucas, \& Smith, 1999; Eid \& Larsen, 2008). As respostas emocionais correspondem a julgamentos de emoções e humores, juntos denominados afetos, feitos pelos próprios indivíduos sobre situaçõesatuais de suas vidas. A satisfação com a vida visa à avaliação de crenças e pensamentos relacionados às suas vidas de maneira geral. Nessa perspectiva, o BES engloba três componentes inter-relacionados que formam um construto global: satisfação de vida geral e afeto positivo e afeto negativo (Diener et al., 1999; Luhmann, Hofmann, Eid, \&

1 Endereço: Rua Ramiro Barcelos, 2600, sala 101, Laboratório de Mensuração, Porto Alegre, RS, Brasil, CEP: 90035-003. E-mail: carwpsi@hotmail.com
Lucas, 2012). Em síntese, o BES refere-se a como as pessoas se sentem e a como avaliam suas vidas.

Um conjunto de fatores pode ser listado como influenciadores e relacionados ao BES, dentre eles os intrínsecos,que são oriundos do próprio indivíduo, como aspectos psicológicos, valores, crenças, religiosidade, estratégias de coping e condições de saúde física. Além dos fatores extrínsecos, tais como aspectos sociodemográficos, culturais e eventos de vida (Woyciekoski, Stenert, \& Hutz, 2012). Porém, ainda há incerteza sobre quais fatores mais impactam o bem-estar, bem como quais são os preditores mais explicativos dentre os comumente citados (Zhang et al., 2009).

\section{Bem-estar subjetivo e características de personalidade}

Há estudos que sugerem que o impacto da personalidade (definida e medida através de distintos modelos) no bemestar é substancial, constituindo-se como um dos seus mais fortes e consistentes preditores (Garcia \& Erlandsson, 2011; Hayes \& Joseph, 2003; Lucas \& Diener, 2008;Steel, Schmidt, $\&$ Shultz, 2008). Por exemplo, Hayes e Joseph (2003) demonstraram que a personalidade explicou de $32 \%$ a $56 \%$ da variância dos escores em BES, dependendo da definição operacional de BES e do tipo de escala utilizada. Em outra 
meta-análise, conduzida por Brajsa-Zganec, Ivanovic e Lipovcan (2011), os fatores de personalidade explicaram $17 \%$ da variância de Satisfação de Vida, 37\% de Afeto positivo e $52 \%$ de Afeto negativo.

Destacam-se entre os estudos que relacionam o BES e personalidade, os que utilizam a perspectiva dos Cinco Grandes Fatores (mais sobre essa abordagem em Hutz et al., 1998). Os fatores Extroversão e Neuroticismo têm sido apontados como os principais relacionados ao BES (Costa \& McCrae, 1980; Diener \& Suh, 1998; Grant, Langan-Fox, \& Anglim, 2009; Lüdtke, Roberts, Trautwein, \& Nagy, 2011; Nunes, Hutz, \& Giacomoni, 2009; Steel et al., 2008). Em estudos longitudinais, Costa e McCrae (1980) concluíram que Extroversão e Neuroticismo predisseram Afeto positivo e Afeto negativo, respectivamente, ao longo de um período de cinco anos. Outros autores também destacaram as associações positivas de Extroversão com Afeto positivo e de Neuroticismo com Afeto negativo (Brajsa-Zganec et al., 2011; Garcia \& Erlandsson, 2011; Hutz, Nunes, Serra, Silveira, \& Anton, 1999; Lucas \& Diener, 2008). A consistência dos achados nesse sentido tem impulsionado pesquisadores a proporemque Extroversão e Neuroticismo oferecem a conexão primária entre personalidade e BES (Diener, Oishi, \& Lucas, 2003; Garcia \& Erlandsson, 2011; Gomez, Krings, Bangerter,\& Grob, 2009).

Outras dimensões dos Cinco Grandes Fatores também demonstram relações significativas com o BES. Num estudo conduzido por Brajsa-Zganec et al. (2011) foram obtidas correlações positivas entre Realização e Afeto positivo. McCrae e Costa (1991) propuseram que Socialização e Realização constituem traços que levam a experiências mais positivas em situações sociais e de conquistas, respectivamente, o que por sua vez, aumenta o BES.

Diener, Suh e Oishi (1997) sugerem uma associação positiva entre Socialização, Realização e BES, e explicam que essa relação pode se dar em função de recompensas ambientais. Por exemplo, pessoas que são sociáveis e com altos escores em Realização podem receber mais reforços positivos do meio e, por isso, experienciar níveis mais altos de BES. Um indivíduo com altos níveis de Realização tende a obter melhores notas, ser melhor remunerado e até mesmo tem maior probabilidade de ter um bom casamento (Donnellan, Conger, \& Bryant, 2004; Ozer \& BenetMartínez, 2006).

Hayes e Joseph (2003) defenderam que Realização constitui um melhor preditor de Satisfação de Vida do que Extroversão. Considerando-se que a Satisfação de Vida abrange elementos cognitivos do bem-estar, é possível esperar que Realização seja um bom preditor dessa dimensão do BES. Realização pode ser um fator de personalidade de destaque para a Satisfação de Vida por refletir uma maior probabilidade das pessoas atuarem de forma mais bem sucedida e conquistarem suas metas (Nunes, Hutz, \& Nunes, 2010).

Os resultados das relações do BES e personalidade permitem pensar que algumas pessoas podem ser mais felizes em função de suas características pessoais e dos recursos internos que dispõem.Além da importante contribuição de traços de personalidade, outros estudos têm enfatizando a investigação do impacto dos Eventos de Vida no BES
(Headey\& Wearing, 1989; Lucas, 2005; 2007a; Luhmann et al., 2012).

\section{Bem-estar subjetivo e eventos de vida}

No que diz respeito às relações entre BES e Eventos de Vida, parece não haver consenso sobre o impacto que os eventos proporcionam ao BES, tampouco se a explicação fornecida pelos mesmos para a variação do BES ultrapassa os efeitos da personalidade. Há pesquisadores que preconizaram uma relação causal entre eventos de vida e afecções psíquicas e sintomatologia médica (o extremo oposto do BES), enquanto outros ressaltam o papel dos traços de personalidade, bem como de outras variáveis, interferindo nessa relação, e até, produzindo os eventos (Garcia, 2007; Headey \& Wearing, 1989; Margis, Picon, Cosner, \& Silveira, 2003; Savoia \& Bernik, 2004).

Segundo Luhmann et al.(2012), a maior parte dos estudos de corte transversal dá suporte à noção de que os EV não têm efeitos duradouros no BES. Ainda, estudos longitudinais sobre as contribuições dos EV no BES têm demonstrado que os efeitos dos eventos podem persistir ao longo de vários anos (para uma revisão, ver Lucas, 2007b). Dentre os achados desses estudos, destaca-se que a reação inicial aos eventos, bem como o índice e tempo de adaptação aos eventos variaram entre os distintos EV. Por exemplo, numa pesquisa conduzida por Lucas, Clark, Georgellis e Diener (2003) a reação inicial ao casamento foi positiva, a adaptação subsequente foi rápida e concluída em até dois anos de casamento. Em outras pesquisas, o tempo necessário para adaptação a eventos, tais como tornar-se deficiente, viúvo ou divorciado, foi maior (Lucas, 2005, 2007a, Lucas et al., 2003). Além disso, Lucas (2007a) observou uma redução nos níveis de satisfação de vida após ocorrência de acidente seguido de deficiência física, não havendo recuperação dos níveis anteriores ao longo de quatro anos.

Headey e Wearing (1989) propuseram que as pessoas podem oscilar nas suas respostas emocionais a eventos, mas acabam retornando a um nível basal de BES, variável em cada pessoa. Os autores sugeriram que a personalidade seria responsável pelos níveis básicos das respostas emocionais, influenciando o BES. Crowley, Hayslip e Hobdy (2003) destacaram que diversos fatores podem interferir na relação entre EV e a possibilidade da ocorrência de estresse subsequente: recursos variados podem amenizar os efeitos do estresse, tais como suporte social, estratégias de coping, além de características de personalidade.

Nesse sentido, Kozma, Stone e Stones (1997) investigaram os fatores que determinam o BES e concluíram que o ambiente, a personalidade e estilos afetivos formam um conjunto que contribui para a estabilidade do BES. Porém, segundo os autores, fatores ambientais estáveis forneceram a menor contribuição e não puderam explicar, isoladamente, a estabilidade em BES.

Se por um lado existem várias evidências que, isoladamente, apontam para fatores de personalidade e eventos de vida influenciando o BES, são poucas as evidências dessas variáveis em conjunto enquanto preditoras do BES. Considerando essa lacuna na área, sobretudo no 
Brasil, e o fato de que estudos anteriores revelam achados por vezes contraditórios acerca da contribuição dessas variáveis para o bem-estar, buscou-se com este estudo: investigar as relações entre fatores de personalidade, eventos de vida e o BES; bem como, testar o poder preditivo dos eventos de vida e de fatores de personalidade para os Afetos positivo e negativo e Satisfação de Vida.

\section{Método}

\section{Participantes}

Participaram 274 estudantes universitários escolhidos por conveniência, com média de idade de 26,7 anos ( $\mathrm{DP}=8,70)$, sendo que $69 \%$ eram mulheres, e todos provenientes de três universidades da região metropolitana de uma capital de Estado da Região Sul do Brasil.

\section{Instrumentos}

Utilizou-se um questionário contendo duas questões sociodemográficas (sexo e idade) e os seguintes instrumentos:

Escala de Afeto Positivo e Afeto Negativo (Watson, Clark, \& Tellegen, 1988; adaptada para o português por Zanon \& Hutz, 2014). A escala é formada por 40 itens distribuídos em dois fatores:afeto positivo e afeto negativo. Cada fator é composto por 20 adjetivos que representam humores e emoções, como, por exemplo: amável, cuidadoso, aflito, impaciente. Os adjetivos são avaliados pelos participantes em uma escala Likert de cinco pontos, em que devem marcar o quanto concordam que cada um deles os descreve adequadamente. Quanto mais próximo do cinco, mais concordam que os adjetivos os descrevem adequadamente. Neste estudo, o coeficiente alfa para o fator Afeto positivo foi de 0,86 e para o fator Afeto negativo foi de 0,91 .

Escala de Satisfação de Vida (Pavot \& Diener, 1993; adaptado para o português por Hutz, Zanon \& Bardagi, 2014). A escala é composta de cinco itens em formato Likert de sete pontos, em que os participantes devem marcar o quanto concordam com cada um deles. Quanto mais próximo do sete, maior o grau de concordância e, consequentemente, a satisfação de vida. Neste estudo, a escala apresentou coeficiente alfa de 0,82 .

Bateria Fatorial de Personalidade(BFP-Nunes et al., 2010). O teste é utilizado para avaliar personalidade a partir do modelo dos Cinco Grandes Fatores de Personalidade. A bateria é composta por 126 itens em formato Likert de sete pontos em que os participantes devem julgar o quanto cada uma das afirmativas se aplica a eles. Os itens abrangem as cinco dimensões da personalidade do modelo: Socialização, 28 itens, refere-se aos tipos de interação que vão da compaixão ao antagonismo, responsividade, empatia, cooperatividade; Realização, 21 itens, verifica o grau de organização, persistência, controle e motivação para alcançar um objetivo; Extroversão, 25 itens, afere níveis de interação interpessoal em termos de quantidade e intensidade, níveis de atividade, necessidade de estimulação; Neuroticismo, 29 itens, avalia o nível de ajustamento emocional e instabilidade, grau de realidade, tolerância à frustração e respostas de coping não adaptativas, níveis de vulnerabilidade, autocrítica e impulsividade, ansiedade e depressão; e Abertura, 23 itens, avalia comportamentos exploratórios e valorização dos mesmos, tal como a convencionalidade. Os coeficientes alfa dos fatores, neste estudo, variaram entre 0,71 para Abertura a experiências a 0,87 para Extroversão.

Lista de Eventos de Vida(Woyciekoski, Natividade, Hutz, \& Laskoski, 2011).Uma lista com 53 eventos de vida selecionados a partir de uma revisão da literatura. São exemplos de eventos: conclusão de curso universitário; demissão; conflitos com o parceiro. Ao lado de cada evento os participantes deveriam indicar se ele ocorreu em suas vidas; e julgar quão intenso positivamente e negativamente foi o evento, em duas escalas distintas de cinco pontos. Para a escala de positividade, quanto mais próximo decinco os participantes marcassem, mais positivo foi considerado o evento; já para a escala de negatividade, quanto próximo o cinco eles assinalassem, mais negativo tinha sido evento para eles.

\section{Procedimentos}

Os participantes foram localizados em duas universidades privadas e uma pública da região metropolitana de uma capital do Estado da região sul do Brasil. Após contatos com os Departamentos e Professores, e explicações sobre os objetivos da pesquisa, procedeu-se com a aplicação coletiva dos questionários nas salas de aula das instituições, em horários cedidos para a pesquisa.O instrumento era preenchido individualmente e os pesquisadores colocavamse a disposição para dirimir dúvidas relacionadas ao preenchimento. A investigação foi conduzida de acordo com as recomendações éticas de pesquisas com seres humanos e obteve aprovação do Comitê de Etica em Pesquisa com Seres Humanos da Universidade Federal do Rio Grande do Sul.

Calcularam-se as médias para Afeto positivo, Afeto negativo e Satisfação de Vida e testaram-se as diferenças de sexo para cada uma delas através de teste $t$ de Student. Também foram conduzidas correlações entre os escores nos domínios do bem-estar (Afetos e Satisfação de Vida), os cinco fatores de personalidade, a avaliação global sobre os eventos de vida (descrito abaixo) e a idade dos participantes através de correlações de Pearson.

A partir do julgamento de quão positivo e quão negativo foram os eventos vivenciados pelos participantes, subtraiu-se da avaliação positiva o valor da avaliação negativa e obtevese o índice de avaliação geral sobre cada um dos eventos ocorridos. Posteriormente calculou-se a média das avaliações dos eventos que ocorreram com cada participante, a fim de caracterizar um índice de avaliação global sobre os eventos de vida. Essas avaliações sobre o evento poderiam variar de -4 , quando a avaliação sobre os eventos foi percebida como muito negativa (ponto 5 na escala de negatividade) e nada positiva (ponto 1 na escala de positividade), a +4 , quando a avaliação sobre os eventos foi considerada muito positiva (ponto 5 na escala de positividade) e nada negativa (ponto 1 na escala de negatividade). 
A partir das respostas à Lista de Eventos de Vida, agruparam-se os participantes entre aqueles que declararam ter vivenciado e os que afirmaram não ter vivenciado cada um dos eventos. Testaram-se, então, as diferenças de médias entre os dois grupos em cada um dos eventos para Afeto positivo, Afeto negativo e Satisfação de Vida através do teste $\mathrm{t}$ de Student. Esse procedimento visou a filtrar, em função das limitações amostrais, o número de eventos vivenciados a serem incluídos nos modelos de predição. Os eventos com diferenças significativas entre os grupos foram incluídos como variáveis independentes (dummy coding: $0=$ não ocorreu o evento, $1=$ ocorreu o evento), juntamente com a avaliação geral sobre eles, nas análises de modelos preditivos para Afeto positivo, Afeto negativo e Satisfação de Vida. Esses modelos foram efetuados através de regressões lineares múltiplas, método stepwise, incluindo-se como variáveis independentes, além das citadas acima: os fatores de personalidade e a avaliação global sobre os eventos. Optouse por esse método de análise a fim de reduzir o número de variáveis no modelo preditivo final, tendo em vista o razoável número de variáveis de entrada nas análises e o tamanho da amostra do estudo.

\section{Resultados}

Inicialmente, computou-se a média dos participantes para cada uma das escalas. Os resultados podem ser vistos na Tabela 1. Também nessa Tabela 1 são mostrados os coeficientes de correlação entre as variáveis do estudo.

Verificaram-se correlações significativas entre Afeto positivo e todos os fatores de personalidade, sendo as mais fortes com Extroversão (positiva) e Neuroticismo (negativa). Afeto negativo correlacionou-se com três fatores de personalidade, mais fortemente com Neuroticismo (positiva). Já a Satisfação de Vida apresentou correlações com quatro fatores de personalidade, três delas de valores similares: Extroversão e Socialização, positivamente e Neuroticismo, negativamente; além de ter serelacionado à Realização.

As avaliações que os participantes fizeram sobre os eventos que vivenciaram foram mais positivas do que negativas, a média de avaliação global foi de $0,13(\mathrm{DP}=0,91)$. Ainda, todos os três domínios do bem-estar correlacionaramse com a avaliação global sobre os eventos, tal que Afeto positivo e Satisfação de Vida de forma positiva e Afeto negativo de maneira negativa. A ocorrência ou não dos eventos de vida mostrou-se discriminador para Afeto positivo

Tabela 1. Médias, Desvios-Padrões e Correlações entre Bem-Estar Subjetivo, Personalidade e Avaliação Global sobre Eventos de Vida

\begin{tabular}{|c|c|c|c|c|c|c|c|c|c|c|}
\hline & $\mathbf{M}$ & DP & 1 & 2 & 3 & 4 & 5 & 6 & 7 & 8 \\
\hline 1- Afeto Positivo & 3,20 & 0,59 & $(0,86)$ & & & & & & & \\
\hline 2- Afeto Negativo & 2,26 & 0,76 & $-0,37 * *$ & $(0,91)$ & & & & & & \\
\hline 3- Satisfação de vida & 4,90 & 1,14 & $0,54^{* *}$ & $-0,36 * *$ & $(0,82)$ & & & & & \\
\hline 4- Extroversão & 4,23 & 0,83 & $0,48^{* *}$ & $-0,14^{*}$ & $0,32 * *$ & $(0,87)$ & & & & \\
\hline 5- Socialização & 5,20 & 0,68 & $0,14^{*}$ & $-0,23 * *$ & $0,33 * *$ & 0,11 & $(0,83)$ & & & \\
\hline 6- Realização & 4,80 & 0,76 & $0,16^{*}$ & 0,02 & $0,18^{* *}$ & $0,14^{*}$ & $0,29 * *$ & $(0,80)$ & & \\
\hline 7- Abertura & 4,56 & 0,66 & $0,19^{* *}$ & $<0,01$ & 0,03 & $0,25 * *$ & $-0,03$ & 0,09 & $(0,71)$ & \\
\hline 8- Neuroticismo & 3,32 & 0,81 & $-0,39 * *$ & $0,58^{* *}$ & $-0,37 * *$ & $-0,20 * *$ & $-0,30^{* *}$ & $-0,22 * *$ & $-0,05$ & $(0,86)$ \\
\hline 9- Avaliação global eventos & 0,13 & 0,91 & $0,24 * *$ & $-0,27 * *$ & $0,27 * *$ & $0,15^{*}$ & 0,05 & $-0,03$ & $-0,03$ & $-0,23 * *$ \\
\hline
\end{tabular}

Nota. $* \mathrm{p}<0,05 ; * \mathrm{p}<0,01$. Os valores da diagonal principal entre parênteses correspondem aos coeficientes alfa das escalas. Avaliação global eventos não é um escore proveniente da subtração entre as avaliações positivas e negativas sobre os eventos de vida que ocorreram com cada participante.

em sete eventos, conforme mostra a Tabela 2. Para Afeto negativo três eventos apresentaram médias diferentes entre aqueles que os vivenciaram e os que não os vivenciaram. Em Satisfação de Vida houve distinção entre os dois grupos para oito eventos.

No modelo preditivo para Afeto positivo, Tabela 3, os fatores de personalidade extroversão e neuroticismo explicaram a maior parte da variância dos dados (30\%), sendo que acréscimos em Extroversão proporcionaram aumentos nos níveis de Afeto positivo e incrementos em neuroticismo contribuíram para diminuir os escores desse Afeto. Para Afeto negativo, Tabela 4, a variável que mais explicou a variância foi o fator neuroticismo (32\%), tal que aumentos nos escores de neuroticismo predisseram níveis mais altos desse Afeto. O modelo para a satisfação de vida, Tabela 5, também apresentou o fator Neuroticismo como o mais explicativo para a variância (13\%); nesse caso, aumentos em neuroticismo acarretaram diminuições em satisfação de vida.

Entre os eventos de vida que as ocorrências explicam o bem-estar, mas com pouca quantidade de variância explicada, destaca-se que ter vivenciado um assalto e ter experimentado um término de relacionamento amoroso proporcionaram incrementos em Afeto positivo. De outro modo, já ter vivenciado a mudança da família para outra cidade diminuíram os escores de Afeto negativo, enquanto ter vivenciado uma doença mental ou física aumentou os níveis de Afeto negativo. Por fim, ter vivido problemas financeiros e um novo emprego reduziram os escores em Satisfação de vida.

\section{Discussão}

\section{Bem-estar eubjetivo e personalidade}

Neste estudo buscou-se contribuir para um maior entendimento acerca do BES, ao se propor a investigação conjunta de variáveis que vem sendo apontadas na literatura como relacionadas ao bem-estar: os cinco grandes 
Tabela 2. Médias e Desvios-Padrões para Afetos Positivo e Negativo e Satisfação de Vida por Grupos de Participantes de Acordo com a Ocorrência do Evento de Vida

\begin{tabular}{|c|c|c|c|c|c|c|c|}
\hline \multirow{3}{*}{ Eventos } & \multicolumn{6}{|c|}{ Ocorrências da Vida } & \multirow[b]{3}{*}{$d$} \\
\hline & \multicolumn{3}{|c|}{ SIM } & \multicolumn{3}{|c|}{ NÃO } & \\
\hline & $n$ & $M$ & $D P$ & $N$ & $M$ & $D P$ & \\
\hline \multicolumn{8}{|l|}{ Afeto Positivo } \\
\hline 7. Obtenção de promoção no trabalho & 76 & 3,32 & 0,58 & 184 & 3,15 & 0,59 & 0,30 \\
\hline 9. Mudança significativa no status financeiro & 142 & 3,26 & 0,56 & 118 & 3,12 & 0,61 & 0,25 \\
\hline 11. Início de relação amorosa/namoro & 235 & 3,24 & 0,56 & 25 & 2,76 & 0,64 & 0,80 \\
\hline 12. Término de relação amorosa/namoro & 206 & 3,26 & 0,57 & 54 & 2,98 & 0,63 & 0,46 \\
\hline 42. Foi vítima de assalto ou sequestro & 88 & 3,34 & 0,53 & 172 & 3,12 & 0,60 & 0,39 \\
\hline 47. Teve doença mental, emocional ou física & 58 & 3,06 & 0,60 & 202 & 3,24 & 0,58 & $-0,29$ \\
\hline 53. Ganhou ou adquiriu bem móvel ou imóvel & 109 & 3,35 & 0,53 & 151 & 3,09 & 0,60 & 0,47 \\
\hline \multicolumn{8}{|l|}{ Afeto Negativo } \\
\hline 30. Família mudou-se para outra cidade & 50 & 2,05 & 0,66 & 213 & 2,32 & 0,77 & $-0,38$ \\
\hline 47. Teve doença mental, emocional ou física & 57 & 2,63 & 0,88 & 206 & 2,16 & 0,69 & 0,60 \\
\hline 53. Ganhou ou adquiriu bem móvel ou imóvel & 108 & 2,13 & 0,72 & 155 & 2,36 & 0,77 & $-0,31$ \\
\hline \multicolumn{8}{|l|}{ Satisfação de vida } \\
\hline 5. Demissão & 73 & 4,61 & 1,17 & 191 & 5,01 & 1,11 & $-0,35$ \\
\hline 6. Novo emprego & 168 & 4,73 & 1,17 & 96 & 5,19 & 1,04 & $-0,41$ \\
\hline 10. Problemas financeiros & 141 & 4,66 & 1,12 & 123 & 5,17 & 1,11 & $-0,46$ \\
\hline 13. Casamento & 58 & 5,18 & 1,01 & 206 & 4,82 & 1,17 & 0,33 \\
\hline 17. Foi rejeitado por homem/mulher que desejava & 137 & 4,72 & 1,12 & 127 & 5,09 & 1,14 & $-0,33$ \\
\hline 27. Aumento discussões/conflitos com familiares/amigos & 147 & 4,73 & 1,15 & 117 & 5,10 & 1,10 & $-0,33$ \\
\hline 47. Teve doença mental, emocional ou física & 58 & 4,58 & 1,23 & 206 & 4,99 & 1,10 & $-0,35$ \\
\hline 53. Ganhou ou adquiriu bem móvel ou imóvel & 110 & 5,13 & 1,04 & 154 & 4,73 & 1,18 & 0,36 \\
\hline
\end{tabular}

Nota. Todas as diferenças são significativas para $p<0,05$. Não são apresentados os eventos cujas diferenças entre ocorrência e não ocorrência não foram significativas.

Tabela 3. Preditores de Afeto Positivo

\begin{tabular}{|c|c|c|c|c|c|c|}
\hline & \multicolumn{6}{|c|}{ Afeto Positivo } \\
\hline & $B$ & $\beta$ & $R 2$ & $\Delta R 2$ & $F(g l)$ & $p$ \\
\hline Constante & 2,38 & & & & & \\
\hline Extroversão & 0,27 & 0,40 & 0,22 & 0,22 & $76,3(1,272)$ & $<0,01$ \\
\hline Neuroticismo & $-0,18$ & $-0,25$ & 0,30 & 0,08 & $59,2(2,271)$ & $<0,01$ \\
\hline 42. Ocorreu assalto ou sequestro & 0,22 & 0,18 & 0,33 & 0,03 & $45,0(3,270)$ & $<0,01$ \\
\hline 12. Ocorreu término de relação amorosa & 0,21 & 0,15 & 0,35 & 0,02 & $37,0(4,269)$ & $<0,01$ \\
\hline Avaliação global sobre eventos & 0,09 & 0,15 & 0,37 & 0,02 & $32,1(5,268)$ & $<0,01$ \\
\hline
\end{tabular}

Nota. Foram testadas como variáveis independentes no modelo: os cinco fatores de personalidade, a avaliação global sobre os eventos de vida e os eventos com diferenças significativas entre aqueles que os vivenciaram (dummy-coding=1) e os que não os vivenciaram e as suas respectivas avaliações gerais. 
Tabela 4. Preditores de Afeto Negativo

\begin{tabular}{|c|c|c|c|c|c|c|}
\hline & \multicolumn{6}{|c|}{ Afeto Negativo } \\
\hline & $\boldsymbol{B}$ & $\beta$ & $R 2$ & $\Delta R 2$ & $F(g l)$ & $p$ \\
\hline Constante & 0,56 & & & & & \\
\hline Neuroticismo & 0,49 & 0,54 & 0,32 & 0,32 & $129,0(1,272)$ & $<0,01$ \\
\hline 47. Ocorreu doença mental, emocional ou física & 0,26 & 0,15 & 0,35 & 0,02 & $71,7(2,271)$ & $<0,01$ \\
\hline 30. Ocorreu mudança da família para outra cidade & $-0,24$ & $-0,13$ & 0,36 & 0,02 & $51,6(3,270)$ & $<0,01$ \\
\hline Realização & 0,14 & 0,14 & 0,38 & 0,01 & $40,7(4,269)$ & $<0,01$ \\
\hline Socialização & $-0,12$ & $-0,11$ & 0,39 & 0,01 & $33,8(5,268)$ & $<0,01$ \\
\hline
\end{tabular}

Nota. Foram testadas como variáveis independentes no modelo: os cinco fatores de personalidade, a avaliação global sobre os eventos de vida e os eventos com diferenças significativas entre aqueles que os vivenciaram (dummy-coding=1) e os que não os vivenciaram e suas respectivas avaliações gerais.

Tabela 5. Preditores da Satisfação da vida

\begin{tabular}{|c|c|c|c|c|c|c|}
\hline & \multicolumn{6}{|c|}{ Afeto Negativo } \\
\hline & $B$ & $\beta$ & $R 2$ & $\Delta R 2$ & $F(g l)$ & $p$ \\
\hline Constante & 4,07 & & & & & \\
\hline Neuroticismo & $-0,34$ & $-0,25$ & 0,13 & 0,13 & $39,9(1,272)$ & $<0,01$ \\
\hline Extroversão & 0,28 & 0,21 & 0,20 & 0,06 & $32,0(2,271)$ & $<0,01$ \\
\hline Socialização & 0,37 & 0,23 & 0,23 & 0,04 & $27,7(3,270)$ & $<0,01$ \\
\hline 10. Ocorreram problemas financeiros & $-0,45$ & $-0,20$ & 0,28 & 0,05 & $26,3(4,269)$ & $<0,01$ \\
\hline $\begin{array}{l}\text { Avaliação geral evento: } 47 \text {. Teve doença mental, } \\
\text { emocional ou física }\end{array}$ & 0,26 & 0,14 & 0,30 & 0,02 & $22,8(5,268)$ & $<0,01$ \\
\hline 6. Ocorreu novo emprego & $-0,26$ & $-0,11$ & 0,31 & 0,01 & $20,0(6,267)$ & $<0,01$ \\
\hline
\end{tabular}

Nota. Foram testadas como variáveis independentes no modelo: os cinco fatores de personalidade, a avaliação global sobre os eventos de vida e os eventos com diferenças significativas entre aqueles que os vivenciaram (dummy-coding=1) e os que não os vivenciaram e suas respectivas avaliações gerais.

fatores de personalidade e eventos de vida. Resultados de correlações mostraram que Afeto positivo está positivamente correlacionado com os fatores Extroversão, Socialização, Realização e Abertura, podendo ser considerada moderada a correlação com Extroversão e fraca com os demais fatores. Esse resultado está de acordo com os encontrados por Diener e Lucas (1999), Hayes e Joseph (2003), McCrae e Costa (1991), Nunes et al. (2009). Além disso, Afeto positivo correlacionou-se negativamente com Neuroticismo, tal como apontaram Costa e McCrae (1980). Quanto à avaliação global dos eventos de vida, houve correlação positiva com Afeto positivo, sugerindo que quanto mais os participantes avaliam como positivos os eventos que ocorreram em suas vidas, maiores os níveis de Afeto positivo.

Afeto negativo correlacionou-se positivamente e moderadamente com o fator Neuroticismo, resultado compatível com os achados da literatura nacional e internacional (Costa \& McCrae, 1980; Haslam, Whelan, \& Bastian, 2009; Nunes et al., 2009). Socialização e Extroversão foram os outros dois fatores de personalidade que apresentaram correlações negativas e fracas, com Afeto negativo, assim como as obtidas no estudo conduzido por Haslam et al. (2009). A avaliação global sobre os eventos de vida mostrou correlação negativa com Afeto negativo, indicando que avaliações mais positivas sobre os eventos que ocorreram na vida relacionam-se com menores pontuações em Afeto negativo.

A Satisfação de Vida correlacionou-se moderadamente com os fatores Extroversão, Socialização e Neuroticismo, com os dois primeiros positivamente, e com o último negativamente; corroborando achados anteriores (Diener \& Lucas, 1999; DeNeve \& Cooper, 1998; Nunes et al., 2009). Ainda se pode observar uma correlação positiva, embora fraca, com o fator Realização, assim como encontraram Brajsa-Zganec et al. (2011) e Hayes e Joseph (2003). Essa correlação poderia ser esperada em função da Realização refletir uma maior probabilidade das pessoas agirem em prol da conquista de suas metas.A correlação da Satisfação de Vida com a avaliação global dos eventos indica que quanto mais positivamente são avaliados os acontecimentos da vida, maior a Satisfação de Vida. Esses resultados são plausíveis, uma vez que Satisfação de Vida envolve uma avaliação positiva da vida. Assim, pessoas satisfeitas com suas vidas, tendem a perceber os eventos que lhes acontecem de forma mais positiva.

Tomados em conjunto, esses resultados em consonância com outros estudos sinalizam a importância dos traços de personalidade, teoricamente estáveis, sobre o BES. Os fatores Extroversão e Neuroticismo destacam-se como os mais fortes influenciadores do bem-estar. Uma tendência a apresentar níveis altos de motivação para buscar estimulação 
em atividades de interação social, que demarca a Extroversão, propicia avaliações positivas sobre o BES; por outro lado, uma propensão a apresentar alterações de humor e vivenciar emoções negativas, que caracteriza o Neuroticismo, acarreta em avaliações negativas sobre o BES. Acredita-se que as relações desses fatores com o BES mostrem-se salientes em função deles serem os fatores mais fortemente associados com estados emocionais, consequentemente esses fatores provocam alterações nas avaliações dos componentes do BES, além de também influenciarem as avaliações sobre a positividade dos eventos de vida.

\section{Bem-estar subjetivo, eventos de vida e personalidade}

Embora se tenham evidências que sugerem que algumas pessoas possam estar mais satisfeitas com a vida e experienciando mais afetos positivos em função de suas personalidades, os resultados deste estudo também demonstram a significativa influência dos eventos de vida sobre o BES. Os três domínios do bem-estar correlacionaramse com a avaliação global dos eventos, tal que Afeto positivo e Satisfação de Vida de forma positiva e Afeto negativo de maneira negativa. Esses achados vão ao encontro da proposição de Diener et al. (1999), de que as interpretações subjetivas das circunstâncias objetivas são mais determinantes do BES do que as circunstâncias em si. Ou seja, a percepção das pessoas sobre o evento, bem como seus recursos para lidar com os mesmos parecem ser aspectos importantes a serem considerados no estudo das relações entre os eventos de vida e o BES.

Por outro lado, no presente estudo, no modelo preditivo do BES, a ocorrência de seis eventos contribuiu mais para explicar o BES do que a avaliação subjetiva dos mesmos. Diante desse achado, e considerando a pequena variância explicada por esses eventos, coloca-se a questão sobre em que medida quatro deles poderiam ser considerados sujeitodependentes (por exemplo: ocorreu término de relação amorosa; ocorreu doença mental, emocional ou física; ocorreram problemas financeiros; ocorreu novo emprego); implicando-se desta forma, a influência da personalidade na produção desses eventos. Sem dúvida, entendemos que mais estudos são necessários a fim de esclarecer essas relações.

No que diz respeito aos modelos preditivos do BES, tem-se que para Afeto positivo as variáveis Extroversão, Neuroticismo, os eventos 'Ocorreu assalto ou sequestro' e 'Ocorreu término de relação amorosa', e a Avaliação global sobre os eventos de vida foram preditores significativos do modelo. Destacando a maior contribuição das dimensões de personalidade para predizer esse Afeto. Incrementos nos níveis de Extroversão proporcionaram aumentos no Afeto positivo; por outro lado, incrementos em Neuroticismo provocaram diminuições nesse Afeto. Os resultados corroboraram os achados de um estudo longitudinal realizado por Costa e McCrae (1980).

Mais especificamente e curiosamente, as ocorrências de 'assalto ou sequestro' e 'término de relação amorosa', constituíram eventos que proporcionaram incrementos no Afeto positivo. Uma possível explicação para o caso de término de relação amorosa poderia ser o fato de tais relações amorosas estarem acarretando alguma forma de sofrimento, e ao seu término consequentes incrementos em Afeto positivo seriam observados. Quanto à ocorrência de assalto ou sequestro, não fica clara a associação, mas uma possibilidade seria o fato de pessoas que passaram por essas experiências terem refletido, revisto seus valores e também as coisas que valorizam em suas vidas, gerando, por consequência Afeto positivo.

É importante destacar que esses resultados não fornecem sustentação a premissa de que eventos considerados positivos pelo imaginário social afetam positivamente o bem-estar, e os negativos, negativamente (Fredrickson \& Losada, 2005; Larsen \& Prizmic, 2008). Aqui temos dois exemplos de eventos que, pelo senso comum, são, de forma geral, considerados negativos, mas ao mesmo tempo sua ocorrênciaproporciona incrementos em Afetopositivo. Isso significa dizer que, coforme também pontuaram Luhmann et al. (2012), em uma meta-analise, que desejabilidade social não parece ser uma categoria útil na investigação dos efeitos dos eventos de vida no BES. Os autores complementaram que não é óbvio, a partir dos eventos, determinar se eles são desejáveis ou não. E mais, adaptação também não é mais demorada para eventos tipicamente considerados indesejáveis (desemprego, por exemplo); e mais rápida para eventos considerados desejáveis (por exemplo, casamento) (Luhmann et al., 2012).

Quanto ao Afeto negativo, o fator de personalidade Neuroticismo mostrou-se a principal variável preditora, conforme pontuaram também Haslam et al. (2009), indicando que quanto maiores os níveis de Neuroticismo, maiores os de Afetonegativo. Outras duas variáveis preditoras de Afetonegativo foram Realização, positivamente, e Socialização, negativamente. Sendo assim, incrementos em Realização geraram um aumento em Afetonegativo. Pessoas com altos níveis de Realização tendem a ser organizadas, persistentes, trabalhadoras, decididas, pontuais, escrupulosas, ambiciosas e perseverantes (Nunes et al., 2010). Com base nessas características, poder-se-ia pensar que a ambição, a persistência e a determinação de pessoas assim possamlhes custar, em algum momento no percurso da realização de suas metas, alguma frustração ou sofrimento.

Incrementos em Socializaçãoproporcionam diminuição dos níveis de Afeto negativo. Essa relação se justifica pelo fato de que pessoas com níveis mais altos em Socialização tendem a ser socialmente agradáveis, calorosas, e possivelmente obtêm suporte social a partir de suas relações, contribuindo para menores níveis de Afeto negativo. A 'ocorrência de doença mental, emocional ou física' também contribuiu para aumentar os níveis de Afeto negativo. Por outro lado, a experiência de 'mudança da família para outra cidade' diminuiu os níveis de Afeto negativo. Uma possível explicação para diminuição dos níveis de Afeto negativo evidenciado com a mudança da família para outra cidade poderia ser o fato de no ambiente familiar existirem conflitos, e na medida em que as pessoas se tornam independentes do seio familiar, minimizam-se os conflitos nas suas relações familiares, proporcionando uma diminuição de Afeto negativo.

Novamente, Neuroticismo comportou-se como principal preditor de Satisfação de Vida, indicando que quanto maiores 
os níveis de Neuroticismo, menor será a satisfação com a vida, um resultado que corrobora achados na literatura (Nunes et al., 2009). Além desse fator, contribuíram para explicar a variância daSatisfação de Vida, os fatores Extroversão e Socialização. As correlações positivas entre as dimensões sugerem que quanto maiores os escores em Extroversão e Socialização, maior a probabilidade de as pessoas serem satisfeitos com suas vidas, o que também corrobora achados recentes com brasileiros e americanos (Hutz et al., 2014).

A ocorrência dos eventos 'problemas financeiros' e 'novo emprego' também constituíram preditores negativos de Satisfação de Vida, sugerindo que a ocorrência de tais eventos contribuiu para a redução dos níveis de Satisfação de Vida. A ocorrência do primeiro evento sugere efeitos da situação econômica no bem-estar das pessoas (ver Minkov, 2009). O fato de 'novo emprego' contribuir para uma redução deSatisfação de Vida pode estar relacionado a dificuldades de adaptação no novo emprego e ansiedade. A avaliação geral do evento 'teve doença mental, emocional ou física' também contribuiu para predizer a Satisfação de Vida, indicando que a forma como os sujeitos avaliam esses eventos, pode interferir na forma como avaliam suas vidas.

Os resultados encontrados neste estudo, juntamente com os de outros pesquisadores supracitados, apontam para personalidade e eventos de vida (ocorrência e avaliação) influenciando o BES. Portanto, ao se buscar compreender o que determina o BES, deve-se considerar que cada indivíduo avalia sua própria vida e vivencia os afetos a partir de recursos internos, como personalidade, mas também em função dos eventos que experiencia, os quais interagem para produzir ou erradicar o bem-estar. Dessa forma, concorrem os fatores intrínsecos e extrínsecos na determinação desse construto (Woyciekoski et al.,2012).Por fim, ressalta-se a necessidade de mais pesquisas que busquem elucidaras relações entre essas variáveis em contextos diversos.

\section{Referências}

Brajsa-Zganec, A., Ivanovic, D., \& Lipovcan, L. K. (2011). Personality traits and social desirability as predictors of subjective well-being. Psihologijske Teme, 20(2), 261-276.

Costa, P., \& McCrae, R. R. (1980). Influence of extraversion and neuroticism on subjective well-being: Happy and unhappy people. Journal of Personality and Social Psychology, 38(4), 668-678. doi: 10.1037/0022-3514.38.4.668

Crowley, B. J., Hayslip, B. J., \& Hobdy, J. (2003). Psychological hardiness and adjustment to life events in adulthood. Journal of Adult Development, 10(4), 237-248. doi: 10.1023/A:1026007510134

DeNeve, K. M., \& Cooper, H. (1998). The happy personality: A meta-analysis of 137 personality traits and subjective well-being. Psychological Bulletin, 124(2),197-229. doi: 10.1037/0033-2909.124.2.197

Diener, E. (1984). Subjective well-being. Psychological Bulletin, 95(3), 542-575. doi: 10.1037/0033-2909.95.3.542

Diener, E., \& Lucas, R. F. (1999). Subjective emotional well-being. In M. Lewis, \& J. M. Haviland (Eds.), Handbook of Emotions (pp. 325-337). New York: Guilford.
Diener, E., Oishi, S., \& Lucas, R. (2003). Personality, culture and subjective well-being: Emotional and cognitive evaluations of life. Annual Review of Psychology, 54, 403-425. doi: 10.1146/ annurev.psych.54.101601.145056

Diener, E., \& Suh, M. E. (1998). Subjective well-being and age: An international analysis. In K. W. Schaie, \& M. P. Lawton (Eds.), Annual Review of gerontology and geriatrics: Focus on emotion and adult development (Vol. 17, pp. 304-324). New York: Springer.

Diener, E., Suh, E. M., Lucas, R. E., \& Smith, H. L. (1999). Subjective well-being: Three decades of progress. Psychological Bulletin, 125(2), 276-302. doi: 10.1037/0033-2909.125.2.276

Diener, E., Suh, E., \& Oishi, S. (1997). Recent findings on subjective well-being. Indian Journal of Clinical Psychology, 24(1), 25-41.

Donnellan, M. B., Conger, R. D., \& Bryant, C. M. (2004). The big five and enduring marriages. Journal or Research in Personality, 38, 481-504. doi: 10.1016/j.jrp.2004.01.001

Eid, M., \& Larsen, R. J. (2008). Ed Diener and the science of subjective well-being. In M. Eid, \& R. J. Larsen (Eds.), The science of subjective wellbeing (pp. 1-11). New York, NY: Guilford Press.

Fredrickson, B. L., \& Losada, M. F. (2005). Positive affect and the complex dynamics of human flourishing. American Psychologist, 60,678-686. doi: 10.1037/0003-066X.60.7.678

Garcia, C. R. (2007). Eventos de vida em pacientes adultos com transtorno de déficit de atenção/hiperatividade (Unpublished doctoral dissertation). Programa de Pós-Graduação em Ciências Médicas, Psiquiatria, Universidade Federal do Rio Grande do Sul, Porto Alegre, RS.

Garcia, D., \& Erlandsson, A. (2011). The relationship between personality and subjective well-being: Different association patterns when measuring the affective component in frequency and intensity. Journal of Happiness Studies, 12(6), 1023-1034. doi: 10.1007/s10902-010-9242-6

Gomez, V., Krings, F., Bangerter, A., \& Grob, A. (2009). The influence of personality and life events on subjective wellbeing from a life span perspective. Journal of Research in Personality, 43, 345-354. doi: 10.1016/j.jrp.2008.12.014

Grant, S., Langan-Fox, J., \& Anglim, J. (2009). The Big Five traits as predictors of subjective and psychological wellbeing. Psychological Reports, 105(1), 205-231. doi: 10.2466/ pr0.105.1.205-231

Haslam, N., Whelan, J., \& Bastian, B. (2009).Big five traits mediate associations between values and subjective well-being. Personality and Individual Differences, 46(1), 40-42. doi: 10.1016/j.paid.2008.09.001

Hayes, N., \& Joseph, S. (2003). Big 5 Correlates of three measures of subjective well-being. Personality and Individual Differences, 34(4), 723-727. doi: 10.1016/S0191-8869(02)00057-0

Headey, D., \& Wearing, A. (1989). Personality, life events, and subjective well-being: Toward a dynamic equilibrium model. Journal of Personality and Social Psychology, 57(4),731-739. doi: 10.1037/0022-3514.57.4.731

Hutz, C. S., Midgett, A., Pacico, J. C., Bastianello, M. R., \& Zanon, C. (2014). The relationshiop of hope, optimism, selfesteem, subjective well-being, and personality in Brazilians and Americans. Psychology, 5, 514-522. doi: 10.4236/ psych.2014.56061 
Hutz, C. S., Nunes, C. H., Silveira, A. D., Serra, J., Anton, M., \& Wieczorek, L. S. (1998). O desenvolvimento de marcadores para a avaliação da personalidade no modelo dos cinco grandes fatores. Psicologia: Reflexão e Crítica, 11(2), 395-411. doi: 10.1590/S0102-79721998000200015

Hutz, C. S., Nunes, C. H. S. S., Serra, J., Silveira, A. D., \& Anton, M. (1999, julho). Relações entre Bem-Estar Subjetivo e Características de Personalidade. Trabalho apresentado no VIII Congresso Nacional de Avaliação Psicológica, Porto Alegre, RS.

Hutz, C. S., Zanon, C., \& Bardagi, M. (2014). Satisfação de Vida. In C. S. Hutz (Ed.), Avaliação em Psicologia Positiva (pp.4348). Porto Alegre: Artmed.

Kozma, A., Stone, S., \& Stones, M. J. (1997). Stability in components and predictors of subjective well-being.Paper presented at the First Conference of the International Society of Quality of Life Studies, Charlotte, North Carolina.

Larsen, R. J., \& Prizmic, Z. (2008). Regulation of emotional wellbeing: Overcoming the hedonic treadmill. In M. Eid,\& R. J. Larsen (Eds.), The science of subjective well-being (pp. 258289). New York, NY: Guilford Press.

Lucas, R.E. (2005). Time does not heal all wounds: A longitudinal study of reaction and adaptation to divorce. Psychological Science, 16(12),945-950. doi: 10.1111/j.14679280.2005.01642.x

Lucas, R. E. (2007a). Long-term disability is associated with lasting changes in subjective well-being: Evidence from two nationally representative longitudinal studies. Journal of Personality and Social Psychology, 92(4), 717-730. doi: 10.1037/00223514.92.4.717

Lucas, R. E. (2007b). Adaptation and the set-point model of subjective well-being: Does happiness change after major life events? Current Directions in Psychological Science, 16, 75-79. doi: 10.1111/j.1467-8721.2007.00479.x

Lucas, R. E., Clark, A. E., Georgellis, Y., \& Diener, E. (2003). Reexamining adaptation and the set point model of happiness: Reactions to changes in marital status. Journal of Personality and Social Psychology, 84, 527-539. doi: 10.1037/00223514.84.3.527

Lucas, R. E., \& Diener, E. (2008). Personality and subjective wellbeing. In O. P. John, R. W. Robins, \& L. A. Pervin (Eds.), Handbook of personality-theory and research (pp. 795-814). New York: The Guilford Press.

Lüdtke, O., Roberts, B. W., Trautwein, U., \& Nagy, G. (2011). A random walk down university avenue: Life paths, life events, and personality trait change at the transition to university life. Journal of Personality and Social Psychology, 101(3), 620-637. doi: $10.1037 / \mathrm{a} 0023743$
Luhmann, M., Hofmann, W., Eid, M., \& Lucas, R. E. (2012). Subjective well-being and adaptation to life events: A metaanalysis. Journal of Personality and Social Psychology, 7, 592-615. doi: 10.1037/a0025948

Margis, R., Picon, P., Cosner, A. F., \& Silveira, R. O. (2003). Stressfull life-events, stress and anxiety. Revista de Psiquiatria Rio Grande do Sul, 25(1), 65-74.

McCrae, R. R., \& Costa, P. T. (1991). Adding liebe und arbeit: The full five-factor model and well-being. Personality and Social Psychology Bulletin, 17(2), 227-232. doi: 10.1177/014616729101700217

Minkov, M. (2009). Predictors of differences in subjective wellbeing across 97 nations. Cross Cultural Research, 43(2), 152-179. doi: 10.1177/1069397109332239

Nunes, C. H. S., Hutz, C. S., \& Giacomoni, C. H. (2009). Associação entre bem estar subjetivo e personalidade no modelo dos cinco grandes fatores. Avaliação psicológica, 8(1), 99-108.

Nunes, C. H. S. S., Hutz, C. S., \&Nunes, M. F. O. (2010). Bateria Fatorial de Personalidade. São Paulo: Casa do Psicólogo.

Ozer, D. J., \& Benet-Martínez, V. (2006). Personality and the prediction of consequential outcomes. Annual Review of Psychology, 57, 401-412. doi: 10.1146/annurev. psych.57.102904.190127

Pavot, W., \& Diener, E. (1993). Review of the Satisfaction with Life Scale. Psychological Assessment, 5, 164-172. doi: 10.1037/1040-3590.5.2.164

Savoia, M. G., \& Bernik, M. (2004). Adverse life events and coping skills in panic disorder. Revista do Hospital de Clínicas da Faculdade de Medicina de São Paulo, 59(6), 337-340.

Steel, P., Schmidt, J., \& Shultz, J. (2008). Refining the relationship between personality and subjective well-being. Psychological Bulletin, 134(1), 138-161. doi: 10.1037/0033-2909.134.1.138

Watson, D., Clark, L.A., \& Tellegen, A. (1988). Development and validation of brief measures of positive and negative affect: The PANAS scales. Journal of Personality and Social Psychology, 54(6), 1063-1070. doi: 10.1037/0022-3514.54.6.1063

Woyciekoski, C., Natividade, J. C., Hutz, C. S., \& Laskoski, L. M. (2011, junho). Eventos de vida avaliados como positivos, negativos e neutros: em busca de um consenso. Pôster apresentado no $V$ Congresso Brasileiro de Avaliação Psicológica, Bento Gonçalves, RS.

Woyciekoski, C., Stenert, F., \& Hutz, C. S. (2012). Determinantes do bem-estar subjetivo. Psico (PUCRS), 43(3), 280-288.

Zanon, C., \& Hutz, C. S. (2014) Escala de Afetos Positivos e Negativos (PANAS). In C. S. Hutz (Ed.) Avaliação em Psicologia Positiva (pp.63-67). Porto Alegre: Artmed.

Zhang, J. P., Yao, S. Q., Ye, M., Huang, H. S., He, G. P., \& Leng, X. H. (2009). A study on the subjective well-being and its influential factors in chronically ill inpatients in Changsha, China. Applied Nursing Research, 22(4), 250-257. 